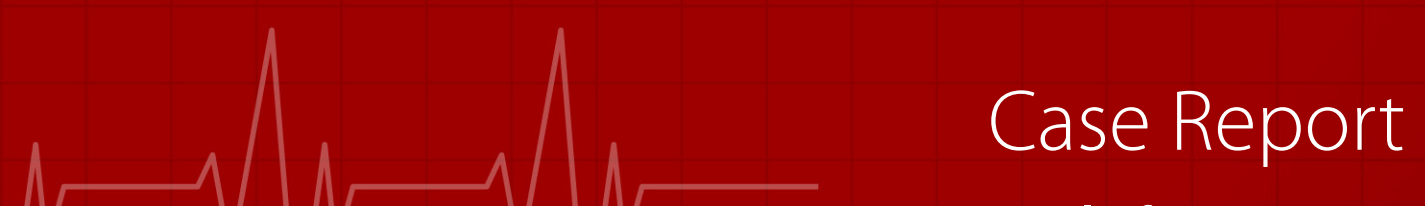

Journal of Emergency Medicine Case Reports

\title{
Two-edged Knife: Massive Pulmonary Embolism and Thrombolytic Contraindication
}

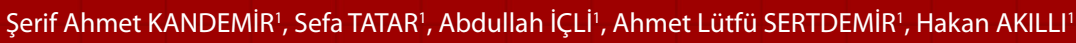

${ }^{1}$ Necmettin Erbakan University, Meram Medical Faculty, Department of Cardiology, Konya, Turkey

\begin{abstract}
Introduction: Pulmonary embolism is a common cause of death among emergency department admissions, and it has a high mortality and morbidity rate. Etiological reasons are generally associated with immobility. Radiological imaging methods are at the forefront in diagnosis. Anticoagulant and thrombolytic therapy may be preferred in treatment according to the hemodynamic condition of the patient.

Case Report: A 56-year-old female patient admitted to the emergency department with sudden onset of dyspnea and syncope with a condition of cardiogenic shock, and echocardiography revealed an enlargement of the right heart chambers and impaired functions, and a tomography was performed with the pre-diagnosis of pulmonary embolism. When systemic thrombolytic therapy was contraindicated in the patient who had embolism on tomography, catheter-based thrombectomy and selective low-dose thrombolytic therapy to the pulmonary artery were administered. The patient, who became hemodynamically stable and his shock condition improved, was discharged with anticoagulant therapy.
\end{abstract}

Conclusion: When left untreated, pulmonary embolism is a disease with a high mortality rate. Although systemic thrombolytic treatments are contraindicated in some patients, successful results can be obtained with locally effective interventional treatments in these patient groups.

Keywords: Pulmonary embolism, catheter-based thrombolysis, thrombolytic therapy.

\section{Introduction}

Pulmonary embolism (PE) is the clinical condition that occurs after thrombus occlusion of the pulmonary artery and/ or its branches. Pulmonary embolism is the third most common cause of death in the USA after myocardial infarction and stroke, and is frequently encountered in emergency room admissions ${ }^{1}$. Mortality in hospitalized patients is as high as $12 \%$ as in outpatients ${ }^{2}$. Genetic and acquired risk factors play a role in the occurrence of PE. Genetic factors are less common and the most common are disorders of the coagulation system. Among the acquired risk factors, conditions such as recent hospitalization history, previous surgical intervention, immobilization, cancer, hormone and steroid therapy (risk 2-3 times higher), pregnancy should be questioned $^{3}$. It should be kept in mind that $30 \%$ of patients with pulmonary embolism do not have any risk factors ${ }^{4}$.

\section{Case report}

A 56-year-old female patient with a history of hemorrhagic cerebrovascular accident (CVA) and cerebral artery aneurysm was admitted to the emergency room with sudden shortness of breath and syncope. Microcoil embolization was performed to the wide necked aneurysm of $10 \mathrm{~mm}$ diameter in the left anterior cerebral artery (ACA) and then to the saccular aneurysm of $5 \mathrm{~mm}$ diameter in the right anterior-inferior cerebellar artery (AICA) by interventional radiology 2 months ago. The patient was evaluated in the emergency department with a blood pressure of 90/60 $\mathrm{mm} / \mathrm{Hg}$, an oxygen-free saturation of $85 \%$, and a pulse rate of $120 /$ min during admission and pH: 7.49, PCO2: 23, PO2: 56, sO2: 89 in blood gas that these values were consistent with pulmonary embolism. On physical examination, the general condition was moderate to poor, tachypnea and anxious. Electrocardiography of the patient had tachycardia (120/ $\mathrm{min})$ and incomplete right bundle branch block. The patient had a troponin height (troponin: 1.27 ug / L ref: 0-0.016). In bedside echocardiography; Right heart cavities were large (Right ventricle (RV) basal diameter: $4.5 \mathrm{~cm}$, D-shape left ventricle) (Figure 1a) and severe impairment in right ventricular systolic functions (RV-Sm: $9 \mathrm{~cm} / \mathrm{s}$ TAPSE: $12 \mathrm{~mm}$ ). Subsequent pulmonary computed tomography (CT) angiography revealed a filling defect consistent with embolism in both pulmonary arteries lobar and segmental branches (Figure 2). In bilateral lower extremity venous doppler ultrasonography for deep vein thrombosis of 
the patient, acute thrombus was observed in the popliteal vein and cranial segments of the deep crural veins. The patient was taken to the cardiology intensive care unit with the diagnosis of massive pulmonary embolism. Thrombolytic therapy was considered an absolute contraindication, after consultation with neurology and neurosurgery, due to the indication for thrombolytic therapy, but had a previous hemorrhagic CVA and cerebral artery aneurysm.It was decided to give pulmonary artery thrombectomy followed by selective thrombolytic therapy to the pulmonary artery in the patient whose general condition was poor, who was hypotensive despite dobutamine $10 \mathrm{mcg} / \mathrm{kg} / \mathrm{min}$ inotropic support and fluid therapy, and who had contraindications for systemic thrombolytic therapy. We opted for catheter-directed low-dose thrombolytic therapy as it was hemodynamically unstable. Tissue-type $10 \mathrm{mg}$ plasminogen activator (tPA) was infused rapidity into the pulmonary artery via a pulmonary arterial catheter (Figure 3). Clot removal performed using a manuel catheter-directed approach. Then, the same catheter was left in the thrombosed proximally pulmonary artery and an additional 16-hour infusion of $10 \mathrm{mg}$ t-PA was administered for 16 hours. While there was a significant improvement in the clinical progress of the patient, the need for inotropes gradually decreased and was discontinued after a while. No neurological adverse events occurred during the 24-hour period. Control echocardiogra- phy was performed on the patient, who had no need for inotropes and achieved significant hemodynamic improvement, regression in right heart chambers (RV basal diameter: $4 \mathrm{~cm}$ ) and improvement in right heart systolic functions (RV-Sm: 16 $\mathrm{cm} / \mathrm{s}$ TAPSE: $14 \mathrm{~mm}$ ) were observed (Figure $1 \mathrm{~b}$ ). The patient, who was followed up in the cardiology intensive care unit for about 3 days, was in good health, hemodynamically stable, and the patient who did not develop any neurological complications, was discharged with a new generation oral anticoagulant treatment (Rivaroxaban15 mg $2 * 1$ ) and was discharged after 3 weeks and a switch to a $20 \mathrm{mg} 1 * 1$ dose of rivaroxaban was planned. The patient was evaluated 3 weeks later in the cardiology outpatient clinic, and a significant clinical and echocardiographic improvement was detected.

\section{Discussion}

In pulmonary embolism, patients may have no symptoms or may present with cardiac arrest or shock. The most common symptoms are resting or exercise dyspnea, chest pain, palpitations, orthopnea, cough, and haemoptysis ${ }^{5}$. Chest pain, which is often of the pleuritic type. Dyspnea is often acute and rapid onset (seconds and minutes) and is more common especially in PE in the main or lobar vessels. In those with
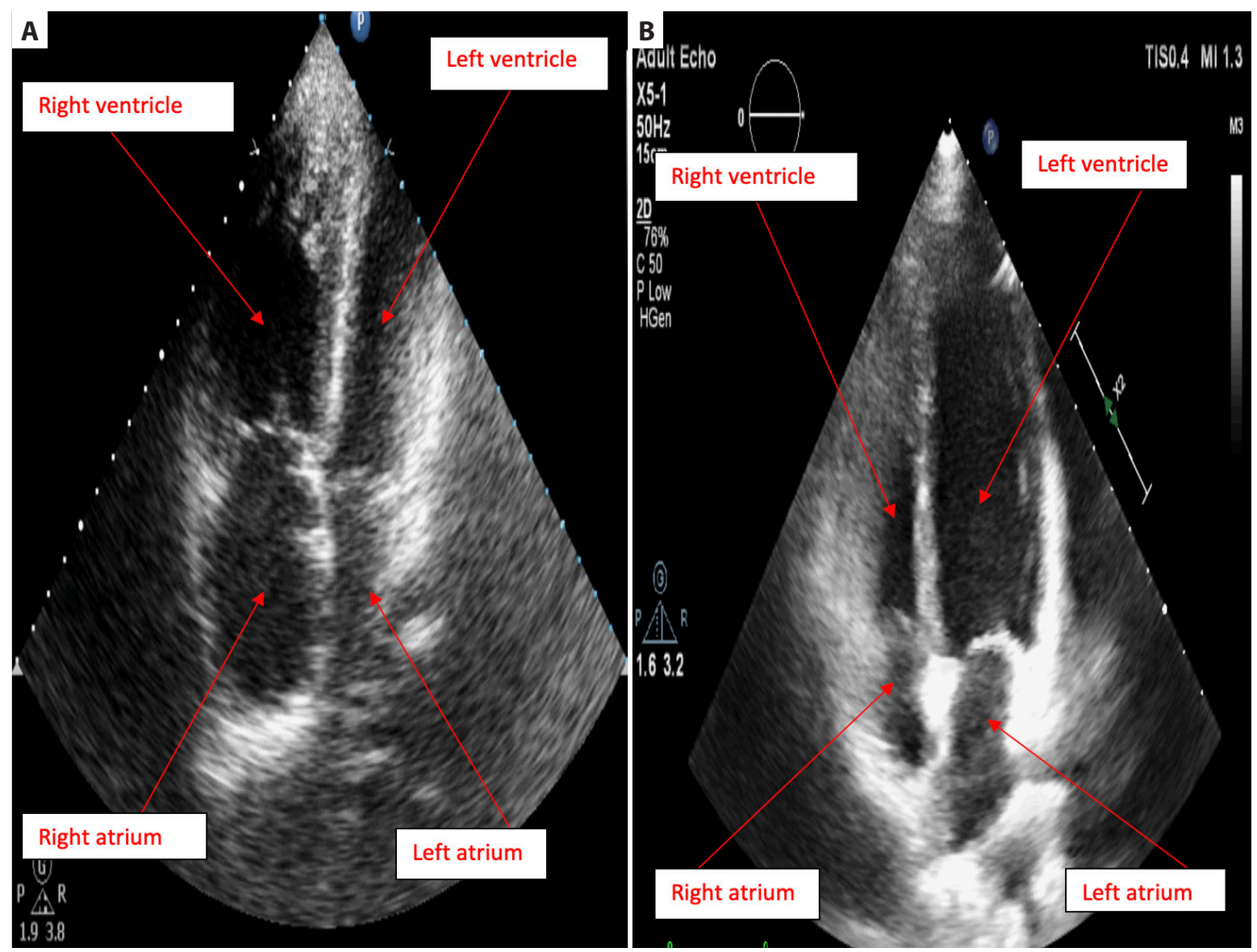

Figure 1: A; Before lytic therapy, the right atrium and right ventricle are markedly dilated. B; After lytic therapy, the right atrium and right ventricle are seen to return to normal sizes. 

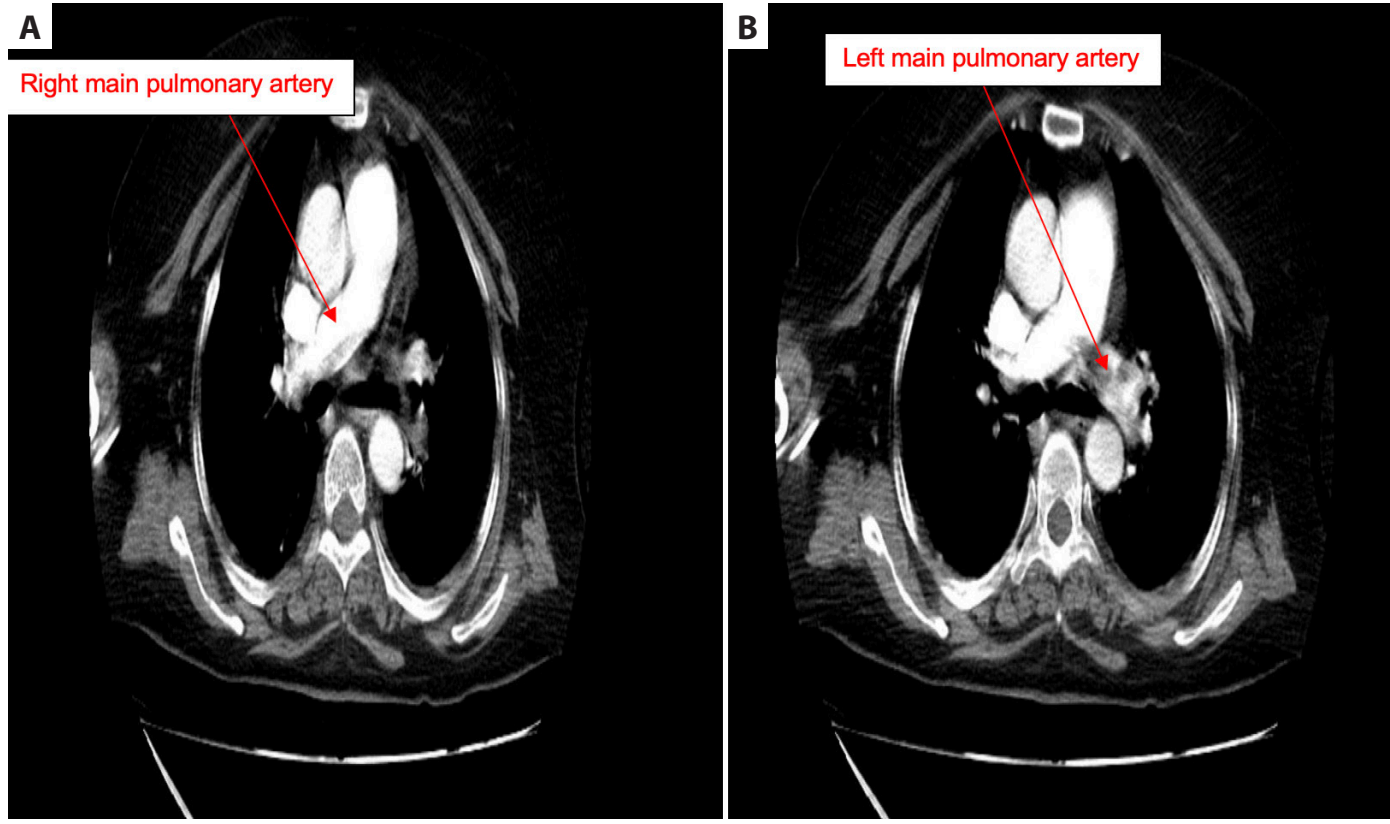

Figure 2: A; Filling defect consistent with embolism is observed in the mid-distal part of the right main pulmonary artery. B; The left main pulmonary artery is completely occluded with thrombus and distal filling is not observed.
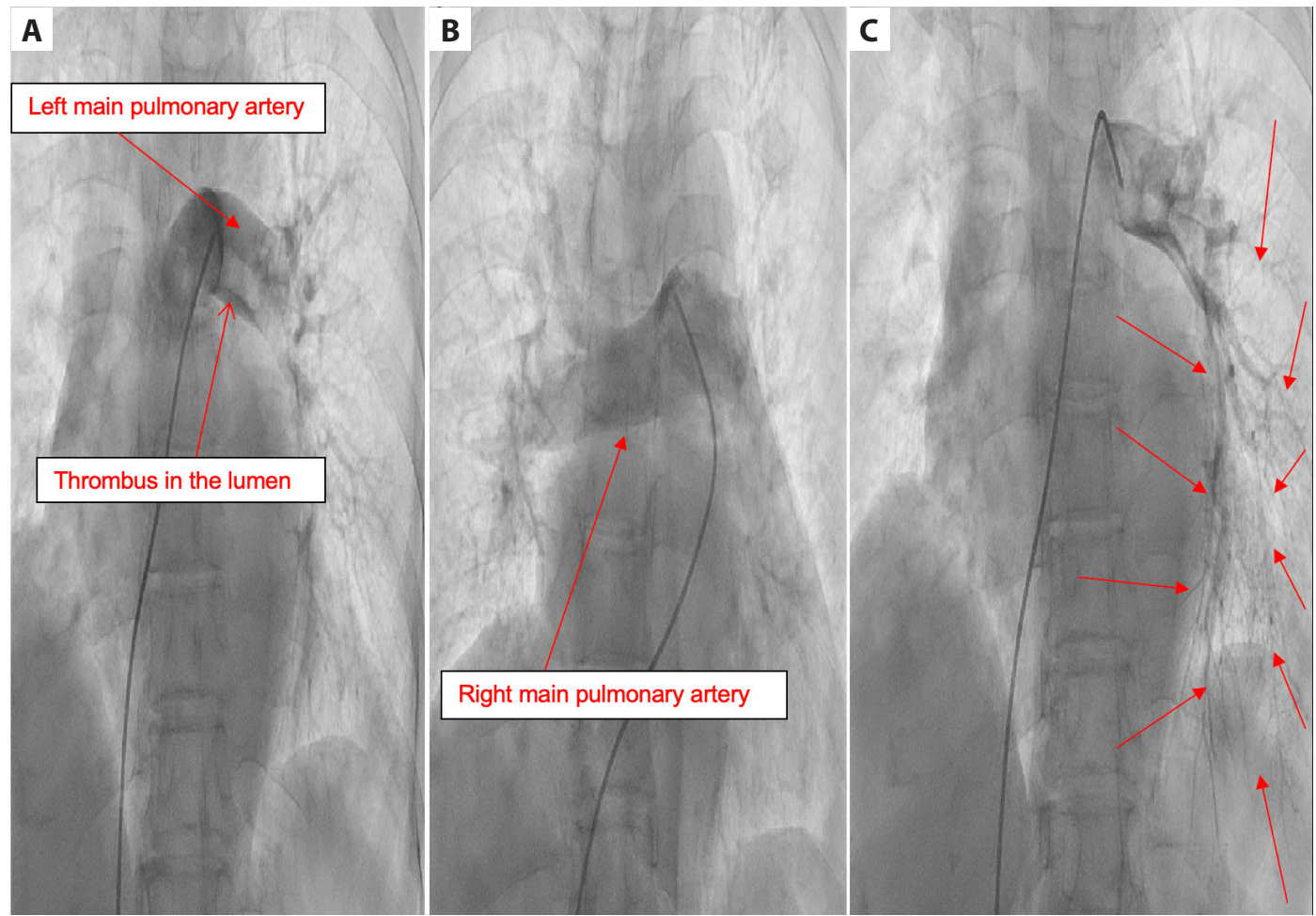

Figure 3: A; Selective pulmonary angiography. Left main pulmonary artery thrombus is completely occluded and distal filling is not observed. B; Filling defect consistent with embolism is observed in the mid-distal part of the right main pulmonary artery and distal weak filling was observed. C; Distal vascular filling was observed after lytic therapy.

heart or lung disease, an increase or worsening of shortness of breath may be the only symptom ${ }^{6}$. Although syncope is seen in less than $10 \%$ of patients, it may be the cause of first presentation ${ }^{7}$. Anamnesis, physical examination, laboratory tests and imaging methods are used in the diagnosis of pulmonary embolism. Pulmonary angiography, which was previously accepted as the gold standard method in detecting pulmonary embolism, has been replaced by pulmonary
CT angiography. In pulmonary embolism, treatment strategy changes according to the clinical condition of the patient and the severity of the embolism. Hemodynamic support and respiratory support constitute the first step in treatment. Anticoagulant therapy and / or thrombolytic therapy can be applied according to risk classification and hemodynamic status. Reperfusion therapy is applied not only with systemic thrombolytic drugs, but also with percutaneous catheter-me- 
diated embolectomy and surgical embolectomy. Systemic or selective thrombolytic therapy can be applied as the first choice in hemodynamically unstable patients or as a rescue therapy in patients who do not show clinical improvement despite anticoagulant therapy.Thrombolytic therapy is more effective than anticoagulant therapy in improving right ventricular function, decreasing pulmonary artery pressure and pulmonary vascular resistance, and improving clinical status. The failure rate of thrombolytic therapy has been reported as $8 \%$, severe bleeding due to its adverse effects, $9 \%$, and intracranial bleeding $7 \%{ }^{8}$. In the presence of contraindications for systemic thrombolytic therapy such as recent surgery, intracranial mass, hemorrhagic cerebrovascular disease, catheter-mediated selective thrombolytic therapy has become a current issue. Mechanical reperfusion is performed by inserting a catheter into the pulmonary arteries through the femoral route, giving low-dose thrombolytics (10-24 mg). Current guidelines emphasize that reperfusion therapy can be given based on echocardiographic findings in a case of pulmonary embolism presenting with shock and hypotension in case of clinical compliance ${ }^{9}$ The success rate considered as hemodynamic stabilization, improvement of hypoxia and discharge is higher and the risk of bleeding is lower than heparin. Systemic thrombolytic therapy has long been used as the first choice in high-risk and haemodynamically unstable patients. Bleeding complications for systemic thrombolytic therapy and the presence of contraindications for its administration have suggested catheter-based therapy (CDT) methods. According to current guidelines, CDT can be applied to high-risk patients for bleeding, unsuccessful systemic thrombolytic therapy and high-risk patients for death without systemic therapy being effective ${ }^{10}$. The guidelines recommend using CDT only in high-risk PE patients but most clinical studies have been conducted in intermediate-risk PE patients. In pulmonary embolism, CDT can be applied mechanically, pharmacologically and in combination of both. In our case we successfully performed mechanical thrombectomy and selective thrombolytic therapy.

\section{Conclusion}

We agree with guidelines which suggest that catheter-directed approaches are options for patients with persistent hemodynamic instability due to PE who are at moderate to high risk of bleeding. Importantly, catheter-directed techniques should be reserved for use in centers with appropriate exper- tise since they are not without risk (eg, further hemodynamic instability, bleeding ). As in our patient, relatively lower tPA doses are now typically administered during CDT, and bleeding rates are now likely in the region of less than 4 percent. It can also be considered that the technique in this trial is different compared to more modern techniques in that the catheter is inserted into the pulmonary artery (proximal to the embolus) rather than "buried" in the embolus.

\section{References}

1. Raskob GE, Angchaisuksiri P, Blanco AN. For the ISTH Steering Committee for World Thrombosis Day. Thrombosis: A major contributor to global disease burden. Arterioscler Thromb Vasc Biol. 2014;34:2363-71.

2. Virk H UI H, Chatterjee S, Sardar P, Bavishi C, Giri J, Chatterjee $\mathrm{S}$. Systemic thrombolysis for pulmonary embolism: Evidence, patient selection, and protocols for management. Intervent Cardiol Clin. 2018;7(1):71-80

3. Crous-Bou $M$, Harrington LB, Kabrhel C. Environmental and genetic risk factors associated with venous thromboembolism. Semin Thromb Hemost 2016;42(8):808-820.

4. Wendelboe AM, Raskob GE. Global burden of thrombosis: Epidemiologic aspects. Circ Res 2016;118:1340-7

5. Arseven $O$, Sevinç C, Ekim N. Pulmoner tromboembolism tanı ve tedavi uzlaşı raporu. Türk Toraks Derneği. 2015.

6. Pollack CV, Schreiber D, Goldhaber SZ. Clinical characteristics, management, and outcomes of patients diagnosed with acute pulmonary embolism in the emergency department: Initial report of EMPEROR (Multicenter Emergency Medicine Pulmonary Embolism in the Real World Registry). J Am Coll Cardiol. 2011;57: 700-6

7. Stein PD, Terrin ML, Hales CA. Clinical, laboratory, roentgenographic, and electrocardiographic findings in patients with acute pulmonary embolism and no pre-existing cardiac or pulmonary disease. Chest. 1991;100:598

8. Marti C, John G, Konstantinides S, Combescure C, Sanchez $O$, Lankeit M, et al. Systemic thrombolytic therapy for acute pulmonary embolism: A systematic review and meta-analysis. Eur Heart J. 2015;36: 605-14.

9. Konstantinides SV, Torbicki A, Agnelli G. ESC guidelines on the diagnosis and management of acute pulmonary embolism. Eur Heart J. 2019;35(43):3033-80.

10. Kucher N, Boekstegers P, Müuller OJ. Randomized, controlled trial of ultrasound- assisted catheter-directed thrombolysis for acute intermediate-risk pulmonary embolism. Circulation. 2014;129:479-86. 